\title{
Immortalized mesenchymal stem cells: a safe cell source for cellular or cell membrane-based treatment of glioma
}

\section{Yuxuan Zhang}

Southern Medical University

Jie Liu

Southern Medical University

Yunzhao Mo

Southern Medical University

Zetao Chen

Southern Medical University

Taoliang Chen

Southern Medical University

Yan Li

Southern Medical University

Yaofeng Zheng

Southern Medical University

Shaokang Deng

Southern Medical University

Xiangdong $\mathrm{Xu}$

Southern Medical University

Huajian Chen

Southern Medical University

Haoqi He

Southern Medical University

Jiansheng Chen

Southern Medical University

Tao Jin

Southern Medical University

\section{Xinlin Sun}

Southern Medical University

Yiquan Ke ( $\nabla$ kyquan@smu.edu.cn )

Southern Medical University https://orcid.org/0000-0002-7356-8559

Jihui Wang 


\section{Research}

Keywords: Immortalization, Human Mesenchymal Stem Cell, Plasma Membrane, Tumour Homing, Drug Delivery, Glioma Therapy

Posted Date: April 12th, 2021

DOl: https://doi.org/10.21203/rs.3.rs-382529/v1

License: (a) (i) This work is licensed under a Creative Commons Attribution 4.0 International License. Read Full License 


\section{Abstract}

BACKGROUND: Mesenchymal stem cells (MSCs) have emerged as putative therapeutic tools due to their intrinsic tumour tropism, anti-tumour, and immunoregulatory properties. The limited passage and selfdifferentiation abilities of MSCs in vitro hinder preclinical studies of mesenchymal stem cells, and makes the MSC-based treatment of tumours lack a stable, uniform, and homogeneous source of cells. In this study, we focused on the safety of immortalised mesenchymal stem cells (im-MSCs) and, for the first time, studied the feasibility of im-MSCs as candidates for the treatment of glioma.

METHODS: The im-MSCs were constructed by the lentiviral transfection of genes, and the proliferative capacity of immortalised MSCs and the proliferative phenotype of MSCs and MSCs co-cultured with glioma cells (U87) were measured using CCK-8 and EdU assays. After long-term culture, karyotype analysis of im-MSCs was conducted. In addition, the tumourigenicity of engineered MSCs was evaluated using soft agar cloning assays. Moreover, the engineered cells were injected into the brain of female BALB/c nude mice. Finally, the cell membranes of im-MSCs were labelled with DIO or DIR to detect their ability to be taken by glioma cells and target in situ gliomas using an IVIS system.

RESULTS: Im-MSCs expressed CD73, CD90, CD105, CD29, and CD44 but did not express CD45, CD34, CD14, CD11b, or CD31. Engineered MSCs maintained the ability to differentiate into mesenchymal lineages in vitro. Im-MSCs showed stronger proliferative capacity than unengineered MSCs without colony formation in soft agar, no tumourigenicity in the brain, and normal chromosomes. MSCs or imMSCs co-cultured with U87 cells showed enhanced proliferation ability, but did not show malignant characteristics in soft agar cloning experiments. Immortalised cells continued to express homing molecules. The cell membranes of im-MSCs were taken up by glioma cells and targeted in situ gliomas in vivo.

CONCLUSIONS: Im-MSCs are promising candidates for cellular anti-glioma therapy. Im-MSCs provide a safe, adequate, quality-controlled, and continuous source of cells or cell membranes for the treatment of glioma.

\section{Background}

Tumours are a common challenge in today's world, especially some refractory tumours, such as glioma. Traditional treatments for glioma include surgery, radiotherapy, and chemotherapy, but the prognosis is still poor[1]. Current efforts are focused on developing targeted molecular therapies, immunotherapies, gene therapies, and novel drug delivery technologies[2]. Cell-based active targeted drug delivery is advantageous because of its inherent targeting capabilities, biocompatibility, and host integration, providing therapeutic efficacy and minimal side effects[3]. Natural drug carriers, such as cells and cell membranes, are very promising for treating tumours. They appear to be complex but can take advantage of the inherent tissue targeting capabilities of specific cell types due to ligand-receptor binding and specific chemokines to achieve actively targeted drug delivery[3], such as CXCR4 and SELP, and SELPLG. 
Stem cells are known to have the ability to self-renew and differentiate. Furthermore, in recent years, studies have suggested that certain stem cells also have tumour homing ability[4]. Mesenchymal stem cells are adult stem cells isolated from various tissues, such as the umbilical cord[5], adipose tissues[6], and bone marrow[7]. Owing to the tumour tropism of stem cells, they are candidates for anti-tumour therapy by carrying drugs or secreting anti-tumour agents, such as engineered mNSCs that secrete tumour necrosis factor apoptosis-inducing ligand to the tumour cavity of glioblastoma (GBM) bearing mice[8]. However, limited passage ability and cell senescence during in vitro culture limit MSCs as a longterm cellular source for tumour therapy. Furthermore, different individuals and tissue-derived MSCs are heterogeneous[9].

Moreover, there are different functional subsets of MSCs from the same organism. It is necessary to immortalize MSCs to obtain a stable, sufficient, and homogeneous cell source or long-acting therapeutic cell. MSCs have an anti-tumour ability from specific sources. In a previous study, Tátrai et al. constructed an immortalized cell line by transfecting a combination of BMI1 and TERT to human adipose-derived MSCs (hADSCs) without significantly perturbing their phenotype or biological behavior[10]. In our study, we used this method to obtain two types of immortalized MSCs (im-MSCs), human umbilical cord MSCs (hUCMSCs) and hADSCs. In previous studies, it was considered that im-MSCs were not tumourigenic[11]; however, the safety of im-MSCs is still in need of adequate clinical research. MSC membranes are safer and more biocompatible than whole MSCs as a drug carrier. The plasma membrane is the main functional part the homing ability of MSCs. The tumour trophic characteristics of MSCs are mainly attributed to the receptor and transmembrane proteins on the membrane. The key ligands involved in MSC homing include chemokine receptors CCR2, CXCL12, and CXCR4 and adhesion ligands, such as SELPLG and SELP, among which CXCR4 is one of the most important molecules mediating the tumour homing of MSCs[12].

We constructed two representative immortalized human MSCs (hMSCs) that overexpressed the TERT gene and BMI1 gene in the present study. The immunophenotype and directed differentiation ability of the cells before and after transfection were examined. The karyotype of immortalized cells was analyzed after long-term culture to understand the gene stability. Furthermore, we thoroughly evaluated the tumourigenicity of im-MSCs in vitro and in vivo, especially in the brain. We also determined the safety of im-MSCs in the treatment of brain tumours, especially glioma, as a cellular drug carrier. Furthermore, we extracted the cell membranes of the im-MSCs and detected their ability to be taken up by glioma cells. Lastly, we tested MSC membranes targeting in situ gliomas to understand their potential, including as drug carriers.

\section{Methods}

\section{Cell isolation and culture}

hUCMSCs were isolated from Wharton's jelly of human umbilical cord by Tissue explants method[5]. hUCMSCs were cultured in Human Umbilical cord mesenchymal stem cell Basal Medium (Cyagen 
Biosciences Inc, USA) containing 10\% fetal bovine serum (Cyagen Biosciences Inc, USA). hADSCs were cultured in Human adipose mesenchymal stem cell Basal Medium (Cyagen Biosciences Inc, USA) containing 10\% fetal bovine serum (Cyagen Biosciences Inc, USA), which were a generous gift from Pro. Hongbo Guo (Southern Medical University, China).

The human GBM cell lines U87 (American Type Culture Collection, USA), U251 (China Academia Sinica Cell Repository, China) were cultured in Dulbecco's modified Eagle's medium (DMEM) containing 10\% fetal bovine serum (Biological Industries, Israel). HAs (ScienCell Research Laboratories, USA) and HEB were grown in 1640 (Gibco, CA, USA) containing 10\% fetal bovine serum (Biological Industries, Israel). The U87-Luc cell line was generated in our laboratory via transfection with a reporter gene encoding firefly luciferase. All cells were grown in a humidified atmosphere of $5 \% \mathrm{CO}_{2}$ at $37^{\circ} \mathrm{C}$.

\section{Immunophenotyping}

Cells were isolated with $0.25 \%$ trypsin contain $0.1 \%$ ethylenediaminetetraacetic acid and washed by PBS (Gibco, CA, USA) three times. The MSCs were cultured with CD11b (PE), CD14 (APC), CD29 (PE), CD31 (FITC), CD34 (PE), CD44 (FITC), CD45 (FITC), CD73 (APC), CD90 (PE) and CD105 (FITC) monoclonal antibodies for 20 minutes at RT, and detected with a BD FACSVerse flow cytometer. The data were analyzed by FlowJo software (BD, USA).

\section{Lentiviral gene transfer}

LV-TERT (pLOV-EF1a-PuroR-CMV-TERT-3FLAG) were purchased from Genechem (shanghai, china). LVBMI1 (pLOV-EF1a-NEO-CMV-BMI1) were purchased from Genepharma (Suzhou, china). Cell culture using $500 \mu \mathrm{l}$ of polybrene-containing $(5 \mu \mathrm{g} / \mathrm{ml})$ medium in 24-well plates, overexpressing Lentivirus were transfected with primary MSCs at multiplicity of infection (MOI) of 50 . Cells were selected and maintained with puromycin (5 $\mathrm{gg} / \mathrm{mL}$, Solarbio, China) or G418 (500 $\mu \mathrm{g} / \mathrm{ml}$, Macklin, Shanghai, China) after transfection for 72 hours.

HA1800 transfected using LV-mCherry-Luc (pLenti-CMV-mCherry-linker-Luc-PGK-Blasticidin, OBiO, Shanghai, China) at MOI of 1 , were selected and maintained with Blasticidin $(10 \mu \mathrm{g} / \mathrm{ml})$. hUCMSC-Luc, hADSC-Luc, im-hUCMSC-Luc and im-hADSC-luc were also obtained by the above method, but different $\mathrm{MOI}$ of 50 .

\section{RNA isolation and RT-PCR}

Total RNA was extracted with TRIzol (Takara, Japan), and the concentration of RNA was measured by using NanoDrop2000 spectrophotometer (Thermo Fisher, USA). 1000 ng of RNA were reverse-transcribed into cDNA by using the PrimeScript ${ }^{\text {TM }}$ RT Reagent Kit with gDNA Eraser (Takara, Japan). Quantitative RTPCR was performed in a CFX96 real-time PCR detection system (Bio-Rad, USA) using SYBR premix (Takara, Japan). All reactions were repeated three times, and human GAPDH was the endogenous control. Primers for detecting gene expression were shown in the Supplementary Table 1.

\section{Western blotting and analysis of MSC membrane proteins}


Western blotting was performed as previous research[13]. After cells lysing with RIPA (CWBio, Beijing, China) on ice for half an hour, the supernatant was centrifuged to obtain total protein. After measuring the protein concentration by the BCA assay, the loading buffer was added to the supernatant and boiled for ten minutes to obtain protein samples. The protein sample was separated in SDS-PAGE gels and the target protein was transferred to the PDVF transfer membranes (Millipore, Germany) according to the guidelines of protein ladder (Thermo Fisher, USA) and the membranes were blocked with 5\% BSA for 1 hour at RT. The membranes were incubated with the primary antibody overnight at $4^{\circ} \mathrm{C}$, washed three times with TBST every ten minutes, and then incubated with the secondary antibody for 1 hour at room temperature (RT) before washing. Visualization of the immunoreactive band using Image Lab (Bio-Rad, USA) after adding luminol-based chemiluminescent substrate (ECL, Millipore, Germany). The band density was analyzed with Image Lab software (Bio-Rad, USA). The antibodies used for detecting proteins expression are shown in the Supplementary Table 2.

\section{Differentiation Studies In Vitro}

Adipocytic Differentiation. MSCs isolated from 6-well plate with trypsin-EDTA, $4 \times 10^{5}$ cells were added to the new culture plate, and the medium consists of $1 \mu \mathrm{M}$ dexamethasone, $10 \mathrm{mg} / \mathrm{L}$ insulin, $0.5 \mathrm{mM}$ IBMX, $200 \mu \mathrm{M}$ Indomethacin was added after 24 hours of liquid exchange. The medium changed every third day during 2-3 weeks. MSCs were fixed in $4 \%$ paraformaldehyde after cells differentiation, stained with oil red 0 . Then, specimens were placed under a microscope (Leica, Germany) and photographed.

Osteogenic Differentiation. The MSCs were seeded in a new 6-well plate in the same way as above, cultured in medium that contain $0.1 \mu \mathrm{M}$ Dexamethasone, $50 \mu \mathrm{g} / \mathrm{L}$ VitC, $10 \mathrm{mM}$ beta-Glycerophosphoric acid disodium salt. The differentiated cells were fixed with $4 \%$ paraformaldehyde and stained with ruthenium, recording with a microscope (Leica, Germany).

Chondroblastic Differentiation. MSC pellets containing $4 \times 10^{5}$ cells were cultured in ChondroDiff Medium (Bgscience, Guangzhou, China) for 2-3 weeks, the medium changed every third day during the period. The medium consists of ITS supplement, TGF- $\beta 3$, ascorbate, proline, dexamethasone, sodium pyruvate, penicillin and streptomycin. The cell pellets were fixed in $4 \%$ paraformaldehyde and subjected to conventional paraffin sectioning. Sections were stained with alcian blue. The images were obtained with microscope (Leica, Germany).

\section{Cell proliferation assay}

Cell counting Kit-8 (CCK8) and EdU assay were used to investigate proliferation. We used a CCK-8 kit (Dojindo, Shanghai, China) to measure proliferation of cells. $100 \mu \mathrm{l}$ of cells suspension containing 1000 cells was added to each well in a 96-well plate and placed in a $37^{\circ} \mathrm{C}$ incubator containing $5 \% \mathrm{CO}_{2}$. After the cells were attached, a mixture of $10 \mu \mathrm{l}$ of CCK-8 reagent and $90 \mu \mathrm{l}$ of medium was added to each well of a 96-well plate, and five replicate wells were repeated. Incubate in a $37^{\circ} \mathrm{C}$ incubator containing $5 \% \mathrm{CO}_{2}$ for 1 to 2 hours, then test the OD450 with a microplate reader (Biotex, USA). 
In addition, the cells were cultured in 96-well plates and treated with $100 \mu \mathrm{l}$ of medium containing $20 \mu \mathrm{M}$ EdU (5-ethynyl-20-deoxyuridine, Keygene, China). After incubating for 2 hours at $37^{\circ} \mathrm{C}$ containing $5 \% \mathrm{CO}_{2}$, the cells were fixed with $4 \%$ paraformaldehyde for 15 minutes, Cell nuclei were stained with DAPI. The rate of proliferation was calculated according to the manufacturer's instructions (KeyFluor488 Click-iT EdU Kit, keygen BioTECH, Jiangsu, China). Finally, images were obtained with an inverted fluorescence microscope (Leica, Germany).

\section{Soft agar assay}

$1.2 \%$ argrose (Macklin, Shanghai, China) solution and $2 \times$ medium were mixed in a ratio of $1: 1$, and $1.5 \mathrm{ml}$ of the mixture was quickly added to each well of a 6-well plate, and the gel was allowed to solidify at RT. Then, $0.7 \%$ argrose and $2 \times$ medium were mixed in $1: 1$ ratio, and cells suspension of 10,000 cells per well was added to the above mixture. The mixture was quickly added to 6 -well plate at $1 \mathrm{ml}$ per well after mixing. After the upper agar was solidified, Plate was placed in $37^{\circ} \mathrm{C}$ incubator with $5 \% \mathrm{CO}_{2}$ for $2-3$ weeks. Repeat three wells for each cell line.

\section{Safety study in vivo}

Mesenchymal stem cells carrying the luciferase gene were injected intracranially or subcutaneously into 4-weeks-old female BALB/c nude mice (Medical Laboratory Animal Center of Southern Medical University, China). After intraperitoneal injection of fluorescein substrate for 10 minutes, in vivo imaging system (IVIS, Lumina II, USA) was used to detect intracellular growth of the cells for 4, 7 and 14 days. U87-Luc was used as a positive reference. HA1800-Luc was used as a negative reference.

\section{Isolation of MSC plasma membranes}

Separation of the plasma membranes (PM) from MSCs by previous methods[14]. Briefly, the cell pellet Isolated from the culture dishes was resuspended in IB-1 buffer $(225 \mathrm{mM}$ mannitol, $75 \mathrm{mM}$ sucrose, 30 $\mathrm{mM}$ Tris- $\mathrm{HCl}, 0.5 \%$ BSA, $0.5 \mathrm{mM}$ EGTA, protease inhibitor cocktail, phosphatase inhibitor cocktail, $\mathrm{pH}$ 7.4) and the cells were lysed with a Ultrasonic breaker, and then centrifuged at $800 \mathrm{~g}$ for 5 mins at $4^{\circ} \mathrm{C}$ to discard the pellet, repeat the previous step to remove nuclei and unbroken cells. Continue to separate the supernatant at $10000 \mathrm{~g}$ for 5 mins at $4^{\circ} \mathrm{C}$ with a centrifuge and discard the pellet. And then centrifuged at $25000 \mathrm{~g}$ for 20 mins at $4^{\circ} \mathrm{C}$ discard the supernatant. The final pellet was dissolved in IB-2 buffer $(225 \mathrm{mM}$ mannitol, $75 \mathrm{mM}$ sucrose, $30 \mathrm{mM}$ Tris-HCl, protease inhibitor cocktail, phosphatase inhibitor cocktail, $\mathrm{pH}$ 7.4), and the concentration measured by BCA method was saved at $-80^{\circ} \mathrm{C}$.

\section{Intracranial glioma xenograft model}

All animal experiments comply with "Guide for the Care and Use of Laboratory Animals" of the National Institutes of Health. U87-luc cells $\left(5 \times 10^{5}\right)$ were injected under the guidance of a stereotaxic instrument to the right frontal lobe of the female BALB/c nude mice (Medical Laboratory Animal Center of Southern Medical University, China) under a general anaesthetic. After the operation was completed, the scalp was sutured and returned to the animal room after the animals have awakened. Tumour growth was measured with the IVIS. 


\section{Experiment of glioma cell uptake MSC cell membranes in vitro}

After extracting the cell membrane, incubate with DIO at RT and protected from light for half an hour, then centrifuge with $25000 \mathrm{~g}, 20$ mins, $4^{\circ} \mathrm{C}$, wash with PBS two times, and obtain DIO-labeled cell membranes stored at $4^{\circ} \mathrm{C}$ protected from light. Add $10 \mu \mathrm{PM}$ (approximately $1 / 5$ of the $10 \mathrm{~cm}$ diameter cell culture dish) to each well of the 12-well plate, and then incubate $0.5 \mathrm{~h} / 2 \mathrm{~h} / 4 \mathrm{~h} / 6 \mathrm{~h} / 8 \mathrm{~h} / 10 \mathrm{~h}$, digested cells and centrifuge with 1000rpm and the pellet wash with PBS. Detection of DIO signals in glioma cells by flow cytometry. PM and glioma cells were incubated together in a confocal dish for 8 hours, fixed with $4 \%$ paraformaldehyde, and then blocked with 5\% BSA at RT for 1 hour. Then incubated with antibody at RT for 1 hour or overnight at $4^{\circ} \mathrm{C}$, followed by incubation with goat anti-rabbit secondary antibody (Alexa Fluor 555, Invitrogen, USA) for $1 \mathrm{~h}$ in a humidified, dark environment. The nuclei were stained with DAPI for 15 mins. Images were obtained with a confocal laser microscope (Leica, Germany).

\section{In vivo tumour-homing assay of MSC plasma membrane}

Cell membranes were extracted by the above method when the cell fusion degree was $80 \sim 90 \%$. DIRlabeled cell membranes were injected into the tail vein of nude mice on the seventh day after planting U87-Luc cells in brain. The distribution of mesenchymal stem cells membranes was measured with the IVIS, and the fluorescence signals were observed at $0.5 \mathrm{~h}, 2 \mathrm{~h}, 6 \mathrm{~h}, 12 \mathrm{~h}, 24 \mathrm{~h}, 48 \mathrm{~h}, 72 \mathrm{~h}$.

\section{Statistical analyses}

Statistical analyses were performed using SPSS IBM 20.0 or GraphPad Prism version 7.0 (GraphPad Software Inc. USA). Data are expressed as mean \pm S.E.M., and differences were considered significant at $P<0.05$. Statistical significance was determined by the t-test or ANOVA.

\section{Results}

\section{Immunophenotype and directed differentiation after immortalization}

The International Society for Cell \& Gene Therapy (ISCT) proposes minimal criteria to define human MSC[15]: 1) MSCs must be plastic-adherent when maintained in standard culture conditions; 2) MSCs must express CD105, CD73, and CD90 and not express CD45, CD34, CD14, CD11b, CD79a, CD19, and HLA-DR surface molecules; and 3) MSCs must differentiate into osteoblasts, adipocytes, and chondroblasts in vitro. In our current study, we first extracted UCMSCs from umbilical cord tissue (Fig. S1A), and flow cytometry showed that im-MSCs and unengineered MSCs all maintained the MSC immunophenotype proposed by the ISCT. High expression of CD105, CD73, and CD90 was detected in primary MSCs (Fig. 1A). In particular, long-term cultured im-hADSCs (P55) and im-hUCMSCs (P30) expressed high levels of CD105, CD70, and CD90. Simultaneously, all MSCs lacked the expression of CD45, CD34, CD14, or CD11b (Fig. 1A). Engineered MSCs maintained the ability to differentiate into 
mesenchymal lineages in vitro, including into osteoblasts (Alizarin Red staining), chondroblasts (Alican blue staining), and adipocytes (Oil Red O staining) (Fig. 1B). These results indicate that im-MSCs conform to the basic definition of MSCs by the ISCT.

\section{Expression of the immortalizing genes}

RT-qPCR and western blotting confirmed the efficient introduction of the immortalizing genes. Engineered MSCs highly expressed TERT and BMI1 mRNA and protein relative to primary MSCs (Fig. 2A, B). Cells morphology did not change after engineering and showed fibroblast-like adherent growth (Fig. 2C).

\section{Cell proliferation and population doubling levels}

The proliferation of engineered cells allowed us to observe the biological processes of im-MSCs transfected with LV-TERT and LV-BMI1. The absorbance measured at $450 \mathrm{~nm}$ represents cell growth and was recorded by a microplate reader. The cell growth curve was significantly increased four days after transfection (Fig. 3A, B). Cell proliferation was detected using an EdU incorporation assay, and the rate of EdU-positive cells was significantly increased after immortalization (Fig. 3C, D). Im-MSCs had a higher proliferative capacity than non-im-MSCs. im-hADSCs could be cultured for more than population doubling levels (PDLs) 60 (Fig. S2A, B) and Im-hUCMSCs could be cultured for more than PDLs 30 (Fig. S2C, D).

\section{Genetic stability}

To determine the genetic stability of engineered MSCs, we performed karyotyping on colchicine-treated im-MSCs after long-term culture. No significant genetic instability was found in the im-hADSC (PDLs55) or im-hUCMSC (PDLs30) (Fig. 4A).

\section{Im-MSCs are safe in vitro and in vivo}

Soft agar (1.2\%) containing double medium was spread on the bottom of the 6-well plate. U87, U251, HA1800, hUCMSC, hADSC, im-hUCMSC, and im-hADSC were mixed separately in $0.7 \%$ soft agar with double medium, and the mixture was spread on $1.2 \%$ soft agar. The mixture was allowed to solidify, and placed on a 6-well plate at $37^{\circ} \mathrm{C}$ in a humidified incubator with $5 \% \mathrm{CO}_{2}$. U87 and $\mathrm{U} 251$ were negative references and HA1800 was a positive reference. After three weeks, the U87 and U251 (Fig. 4B) groups had cell-cloned spheres formed under the microscope, whereas the HA1800 negative reference group showed no sphere formation (Fig. 4B). No clones were formed in either hUCMSCs, hADSCs, im-hUCMSCs, or im-hADSCs (Fig. 4B). Therefore, we believe that im-hUCMSCs and im-hADSCs were not tumourigenic in vitro.

Im-hMSCs were transduced with lentiviral constructs pLenti-CMV-mCherry-linker-Luc-PGK-Blasticidin, sorted, and screened for mCherry fluorescent protein and firefly luciferase (Fluc) expression.

Simultaneously, HA1800 cells were transduced with LV-mCherry-Luc as negative cells. U87-Luc was constructed as a positive reference (Fig. S3). The engineered cells were injected into the cranium of 
female BALB/c nude mice $(n=5)$. The growth of the cells in the body was observed using the IVIS after intraperitoneal injection of D-Luciferin potassium salt $(150 \mu \mathrm{g} / \mathrm{g}$, PerkinElmer, Waltham, Massachusetts, U.S.A.) for 10 mins. The growth of the graft in the brain of mice was recorded on the 4th, 7th, and 14th day after implantation (Fig. 5A). U87-Luc grew in the brain, and eventually led to the death of the mice. However, HA1800, MSC, and im-MSC slowly disappeared after a period of survival in the brain (Fig. 5A). Two weeks after the injection of immortalized UCMSCs under the skin of mice, no abnormal tissues were found (Fig. 5B). Moreover, there were no abnormal tissues in the brain planting site after HE staining (Fig. 5C).

\section{Co-cultivation of U87 and MSC}

The indirect co-incubation of U87 and MSC in 0.4- $\mu \mathrm{m}$ Transwell plates for $48 \mathrm{~h}$ enhanced the proliferation capacity of MSCs (Fig. 4C). However, the co-cultured MSCs could not form clonal spheres in the soft agar colony formation assay (Fig. 4D).

Im-MSC membranes can be taken up by glioma cells without affecting the proliferation of glioma cells in vitro

We studied the potential of im-MSCs membranes as drug carriers for treating glioma by extracting and observing the cell membranes under an electron microscope to a diameter of approximately $100-150 \mathrm{~nm}$ (Fig. 6A), which was beneficial for passing through the blood-brain barrier. SDS-PAGE gel analysis revealed that the protein profiles of the plasma membranes were distinct from those of whole-cell lysates (Fig. 6B). At the same protein concentration, the plasma membrane contained more ATP1A1 protein and less $\beta$-actin and GAPDH. The former was located on the cell membrane, whereas the latter two proteins were in the cytoplasm (Fig. 6C). The key link in the cell membrane drug delivery system is the ability to be taken up by tumour cells. After the DIO-labeled cell membrane was added to the medium, the cell membrane was ingested by glioma cells, as detected by confocal microscopy and flow cytometry (Fig. 7A, B).

\section{Im-MSCs maintain the expression of homing molecules on the cell membranes}

We extracted RNA from every five generations of MSCs and im-MSCs during long-term culture to detect the long-term expression of homing-related molecules. We detected the expression of homing molecules by qPCR. Molecules associated with MSC homing included the chemokine receptor CXCR4 and adhesion ligands, such as PSGL-1 (SELPLG). Membrane proteins were the main determiners of MSC homing behavior. In MSCs, the expression of these molecules after long-term culture was maintained, as detected by qPCR (Fig. 7C). Interestingly, the expression level of SELPLG in im-MSCs was higher than that in unengineered MSCs in some PDLs. Therefore, long-term stable expression of homing molecules supports im-MSCs as cellular or cell membrane-based drug carriers. 


\section{Immortalized mesenchymal stem cell membranes remain ability of tumour-tropic to in site glioma in vivo}

MSC membranes were extracted and labeled with DIR and injected into tumour-bearing mice through the tail vein to detect the in vivo targeting ability of MSC membranes to glioma. The fluorescence signals were observed at $0.5,2,6,12,24,48$, and $72 \mathrm{~h}$ (Fig. 7D). The accumulation peaked $24-48 \mathrm{~h}$ after administration and then decreased gradually (Fig. 7E). Immortalized cell membranes can be used as drug carriers to help the drugs reach and accumulate in the glioma, thereby exerting therapeutic effects.

\section{Discussion}

CAR-T cell therapy opened up new fields for the treatment of tumours. CAR-T therapy is often used in cancers of the blood system; however, for solid tumours[16, 17], especially gliomas[18], it is not effective and a new method of cell therapy needs to be discovered. Stem cells have received increasing attention owing to their unique natural homing ability. More and more clinical trials of MSCs have been approved, but the long-term safety of MSCs remains a concern. Researchers have constructed engineered MSCs that contain a suicide system based on an inducible caspase-9 protein[19]. This system can direct MSC killing to provide the necessary safety by a chemical inducer of dimerization in vivo. But the system requires a complicated construction process, and because of the limited passage capacity of MSC, the system needs to be repeatedly constructed. The above reasons limit the application of the system. In the application of MSCs to the treatment of tumours, one of the most serious problems is the tumourigenicity of MSCs, especially the im-MSCs constructed by lentivirus transfection, which has a stronger proliferative capacity than ordinary MSCs. However, the stronger in vivo viability of im-MSCs allows them to secrete anti-tumour substances longer. In theory, a longer secretion time of cellular anti-tumour agents means longer effects and longer dosing cycles. Based on this point, im-MSCs are an attractive choice for cancer treatment, but their application is based on the premise that it is safe. In our study, we observed that imMSCs do not have tumourigenicity either in vivo or in vitro. In particular, we investigated its safety as a carrier for gliomas. The potential tumourigenicity of im-MSCs implicates them as cell sources for longterm cell therapy, especially for diseases that require long-term maintenance treatment.

Immortalization provides more cells, but the use of a lentivirus as an engineering tool may hinder its clinical translation. The use of other gene transfer pathways in future studies, such as electroporation, or non-genetic pathways, such as repeated transfection of recombinant proteins containing cell-penetrating peptides, may allow their clinical application. Although im-MSCs are not tumourigenic, their biological behavior in vivo, especially in the tumour microenvironment and their interactions with tumour cells, still needs to be considered. In short, MSCs still have many problems as whole-cell drug carriers, and an effective method in the future should be to screen out a subset of MSCs with anti-tumour effects and immortalize them as whole-cell drug carriers. This way, the adverse effects caused by the heterogeneity of MSCs are avoided. Screening and immortalizing anti-tumour subgroups could give full play to the 
production of synergistic anti-tumour effects with drugs by anti-tumour subsets as far as possible. Another strategy is to use stem cell products to load drugs to exert anti-tumour effects.

Other natural drug carriers, such as cell membranes and extracellular vesicles (EVs), have advantages as drug carriers[20] as they have better biocompatibility and biodegradability than chemically synthesized drug carriers[21]. Natural materials have lower toxicity and immunogenicity than chemically synthesized nanocarriers[22]. The types of drugs delivered by EVs mainly include siRNA, miRNA, and small-molecule compounds. Glioblastoma-bearing mice have been treated with exosomes derived from MSCs with high expression of miR-146b, which has anti-cancer effects[23]. The same strategy uses MSC-derived EVs to deliver inhibitors of miRNA that promote cancer, thereby inhibiting tumour progression, such as miR-9[24]. EVs were injected with a small-molecule drug, JSI-124, into a mouse model of glioblastoma and showed tumour-suppressive effects[25]. However, EVs have low acquisition rates, low drug loading, and timeconsuming preparation processes, which limit their application in the clinic. Im-MSCs provide abundant exosome sources for clinical application because of their strong multiplication ability and long passage number. In particular, after immortalizing MSCs that inhibit tumours, they can be used as a source of cells for exosomal vectors, which may achieve better anti-tumour effects than exosomes from unfiltered MSCs. Cell membranes can be an alternative source of EVs and serve as carriers for anti-tumour drugs because of their easy acquisition and less time-consuming preparation processes. At the molecular level, MSC homing involves chemokine receptors CCR2, CXCL12, and CXCR4 and adhesion ligands, such as PSGL-1 and SLeX[12]. Among these molecules, CXCR4 plays a key role in the tumour-tropic processes of MSCs. The sustained expression of these molecules is essential for the use of complete MSCs and MSC membranes as therapeutic carriers. Moreover, most of these molecules are membrane proteins, which further indicates the potential of MSC membranes as therapeutic carriers. In our research, the immortalized cells also maintain the continuous expression of these key molecules, which also provides the molecular basis for the use of immortalized mesenchymal stem cell membranes for tumour homing.

\section{Conclusion}

Im-MSCs maintain the immunophenotype and induce the differentiation of MSCs. The engineered MSCs showed no tumourigenicity in vitro or in vivo, especially when injected directly into the brain, and no tumour formation was observed; thus, MSCs are long-term cells sources for the treatment of brain tumours by homing. Moreover, the cells membranes of im-MSCs showed a homing ability to gliomas; therefore, im-MSC membranes are a potential candidate for anti-tumour drug delivery vehicles. In the future, MSC-based regimens may augment current treatment modalities in glioma.

\section{Declarations}

\section{Acknowledgements}

We express our appreciation to central laboratory of Zhujiang Hospital for laboratory equipment. 


\section{Funding}

This work was supported by National Natural Science Foundation of China (No. 81772651, 82072762, 81772652 and 81802481), Natural Science Foundation of Guangdong Province (No. 2018A030310423), China Postdoctoral Science Foundation funded project (No. 2020M672737) and President Foundation of ZhuJiang Hospital, Southern Medical University (No. yzjj2018rc04).

\section{Authors' contributions}

Yuxuan Zhang: conception and design, collection and/or assembly of data, data analysis and interpretation, manuscript writing, final approval of manuscript. Jie Liu: conception and design, collection and/or assembly of data. Yunzhao Mo, Zetao Chen, Taoliang Chen, Yan Li, Yaofeng Zheng, Shaokang Deng, Xiangdong Xu, Huajian Chen, Haoqi He, Jiansheng Chen, Tao Jin: collection and/or assembly of data. Xinlin Sun: conception and design, financial support, final approval of manuscript. Jihui Wang: conception and design, financial support, final approval of manuscript. Yiquan Ke: conception and design, financial support, administrative support, final approval of manuscript.

\section{Availability of data and materials}

Data sharing not applicable to this article as no datasets were generated or analyzed during the current study.

\section{Ethics approval and consent to participate}

Human UC was obtained from term deliveries (38-40 weeks) after written informed consent, in accordance with the standards of the ZhuJiang Hospital Ethics Committee and with the Helsinki Declaration of 1975, as revised in 1983.

\section{Consent for publication}

Not applicable.

\section{Competing interests}

The authors declare that the research was conducted in the absence of any commercial or financial relationships that could be construed as a potential conflict of interest.

\section{Abbreviations}

MSC, mesenchymal stem cells; im-MSC, immortalised mesenchymal stem cells; IVIS, in vivo imaging system; GBM, glioblastoma; hADSCs, human adipose-derived MSCs; hUCMSCs, human umbilical cord MSCs; RT, room temperature; PM, plasma membrane; EVs, extracellular vesicles; ISCT, International Society for Cellular Therapy; PDL, population doubling level; 


\section{References}

1. Meyer MA. Malignant gliomas in adults. N Engl J Med. 2008;359(17):1850. author reply 1850.

2. Lapointe S, Perry A, Butowski NA. Primary brain tumours in adults. The Lancet. 2018;392(10145):432-46.

3. Thanuja MY, Anupama C, Ranganath SH. Bioengineered cellular and cell membrane-derived vehicles for actively targeted drug delivery: So near and yet so far. Adv Drug Deliv Rev. 2018;132:57-80.

4. Stuckey DW, Shah K. Stem cell-based therapies for cancer treatment: separating hope from hype. Nat Rev Cancer. 2014;14(10):683-91.

5. Li T, Xia M, Gao Y, Chen Y, Xu Y. Human umbilical cord mesenchymal stem cells: an overview of their potential in cell-based therapy. Expert Opin Biol Ther. 2015;15(9):1293-306.

6. Gronthos S, Franklin DM, Leddy HA, Robey PG, Storms RW, Gimble JM. Surface protein characterization of human adipose tissue-derived stromal cells. J Cell Physiol. 2001;189(1):54-63.

7. Campagnoli C, Roberts IA, Kumar S, Bennett PR, Bellantuono I, Fisk NM. Identification of mesenchymal stem/progenitor cells in human first-trimester fetal blood, liver, and bone marrow. Blood. 2001;98(8):2396-402.

8. Kauer TM, Figueiredo JL, Hingtgen S, Shah K. Encapsulated therapeutic stem cells implanted in the tumor resection cavity induce cell death in gliomas. Nat Neurosci. 2011;15(2):197-204.

9. Assoni A, Coatti G, Valadares MC, Beccari M, Gomes J, Pelatti M, Mitne-Neto M, Carvalho VM, Zatz M. Different Donors Mesenchymal Stromal Cells Secretomes Reveal Heterogeneous Profile of Relevance for Therapeutic Use. Stem Cells Dev. 2017;26(3):206-14.

10. Tatrai P, Szepesi A, Matula Z, Szigeti A, Buchan G, Madi A, Uher F, Nemet K. Combined introduction of Bmi-1 and hTERT immortalizes human adipose tissue-derived stromal cells with low risk of transformation. Biochem Biophys Res Commun. 2012;422(1):28-35.

11. Gong M, Bi Y, Jiang W, Zhang Y, Chen L, Hou N, Liu Y, Wei X, Chen J, Li T. Immortalized mesenchymal stem cells: an alternative to primary mesenchymal stem cells in neuronal differentiation and neuroregeneration associated studies. J Biomed Sci. 2011;18:87.

12. Griffith JW, Sokol CL, Luster AD. Chemokines and chemokine receptors: positioning cells for host defense and immunity. Annu Rev Immunol. 2014;32:659-702.

13. Lin J, Ding S, Xie C, Yi R, Wu Z, Luo J, Huang T, Zeng Y, Wang X, Xu A, et al. MicroRNA-4476 promotes glioma progression through a miR-4476/APC/beta-catenin/c-Jun positive feedback loop. Cell Death Dis. 2020;11(4):269.

14. Suski JM, Lebiedzinska M, Wojtala A, Duszynski J, Giorgi C, Pinton P, Wieckowski MR. Isolation of plasma membrane-associated membranes from rat liver. Nat Protoc. 2014;9(2):312-22.

15. Dominici M, Le Blanc K, Mueller I, Slaper-Cortenbach I, Marini F, Krause D, Deans R, Keating A, Prockop D, Horwitz E. Minimal criteria for defining multipotent mesenchymal stromal cells. The International Society for Cellular Therapy position statement. Cytotherapy. 2006;8(4):315-7. 
16. Newick K, O'Brien S, Moon E, Albelda SM. CAR T Cell Therapy for Solid Tumors. Annu Rev Med. 2017;68:139-52.

17. Ma S, Li X, Wang X, Cheng L, Li Z, Zhang C, Ye Z, Qian Q. Current Progress in CAR-T Cell Therapy for Solid Tumors. Int J Biol Sci. 2019;15(12):2548-60.

18. Bagley SJ, Desai AS, Linette GP, June CH, O'Rourke DM. CAR T-cell therapy for glioblastoma: recent clinical advances and future challenges. Neuro Oncol. 2018;20(11):1429-38.

19. Ramos CA, Asgari Z, Liu E, Yvon E, Heslop HE, Rooney CM, Brenner MK, Dotti G. An inducible caspase 9 suicide gene to improve the safety of mesenchymal stromal cell therapies. Stem Cells. 2010;28(6):1107-15.

20. Kooijmans SAA, Schiffelers RM, Zarovni N, Vago R. Modulation of tissue tropism and biological activity of exosomes and other extracellular vesicles: New nanotools for cancer treatment. Pharmacol Res. 2016;111:487-500.

21. Ingato D, Edson JA, Zakharian M, Kwon YJ. Cancer Cell-Derived, Drug-Loaded Nanovesicles Induced by Sulfhydryl-Blocking for Effective and Safe Cancer Therapy. ACS Nano. 2018;12(9):9568-77.

22. Tong Q, Qiu N, Ji J, Ye L, Zhai G. Research Progress in Bioinspired Drug Delivery Systems. Expert Opin Drug Deliv. 2020;17(9):1269-88.

23. Katakowski M, Buller B, Zheng X, Lu Y, Rogers T, Osobamiro O, Shu W, Jiang F, Chopp M. Exosomes from marrow stromal cells expressing miR-146b inhibit glioma growth. Cancer Lett. 2013;335(1):201-4.

24. Munoz JL, Bliss SA, Greco SJ, Ramkissoon SH, Ligon KL, Rameshwar P. Delivery of Functional AntimiR-9 by Mesenchymal Stem Cell-derived Exosomes to Glioblastoma Multiforme Cells Conferred Chemosensitivity. Mol Ther Nucleic Acids. 2013;2:e126.

25. Wang J, Dong Y, Li Y, Li W, Cheng K, Qian Y, Xu G, Zhang X, Hu L, Chen P, et al. Designer Exosomes for Active Targeted Chemo-Photothermal Synergistic Tumor Therapy. Adv Func Mater. 2018;28(18):1707360.

\section{Figures}


A

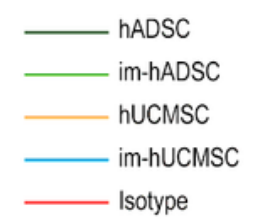

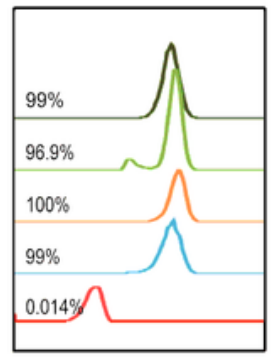

CD29

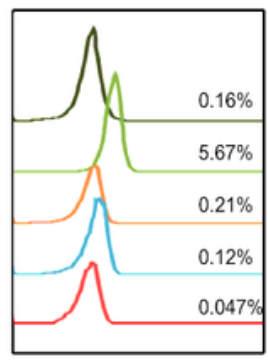

CD11b

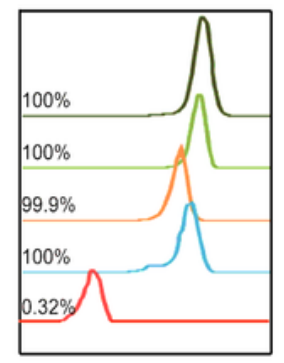

CD44

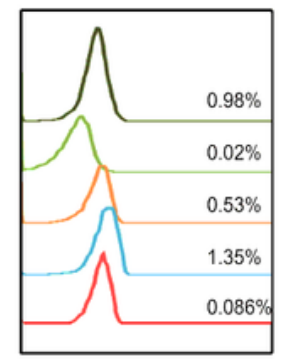

CD14

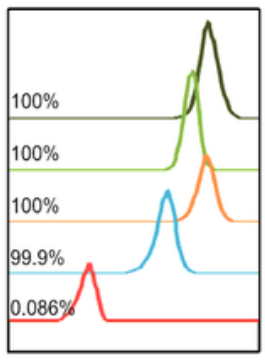

CD73

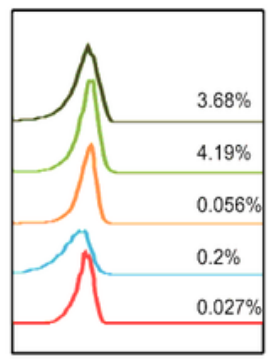

CD31

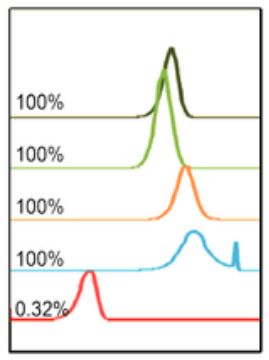

CD90

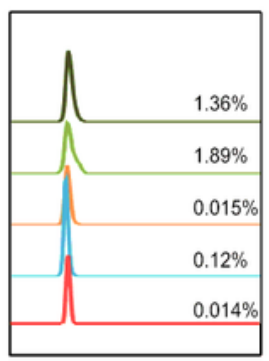

CD34

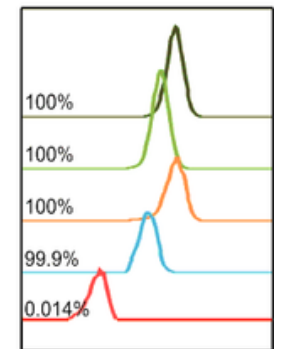

CD105

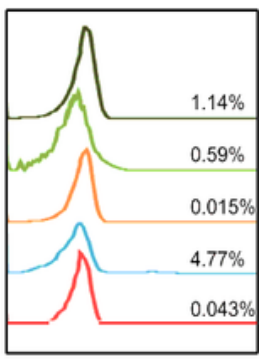

CD45

B

osteoblasts
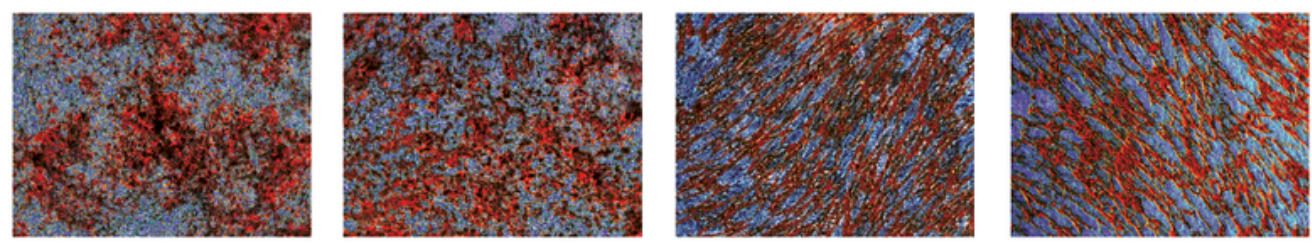

adipocytes
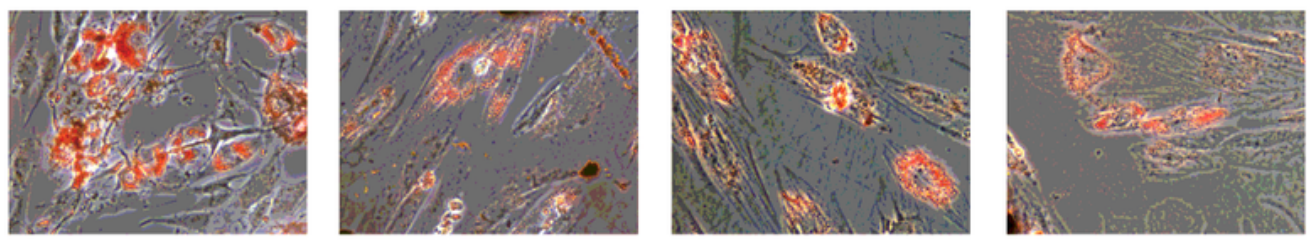

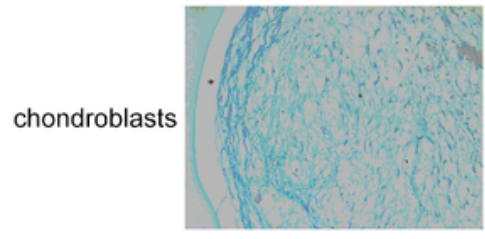

hADSC

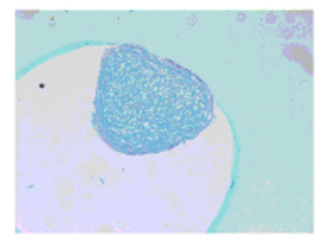

im-hADSC

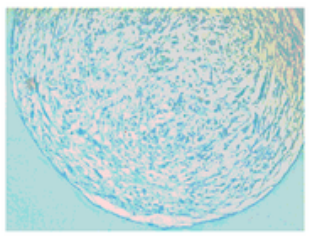

hUCMSC

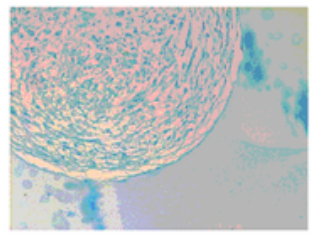

im-hUCMSC

Figure 1

Immunophenotype and directed differentiation after immortalization. (A) Flow cytometry showing surface markers of mesenchymal stem cells from hADSC and hUCMSC. Immortalization of mesenchymal stem cells did not change the immune- phenotype of mesenchymal stem cells. (B) As defined by ISCT, hADSC, hUCMSC, and immortalized mesenchymal stem cells all have the ability to differentiate into osteoblasts, adipocytes and chondroblasts, magnification, $\times 100, \times 400, \times 40$, respectively. 
hADSC, human adipose-derived mesenchymal stem cells; hUCMSC, human umbilical cord mesenchymal stem cells; ISCT, International Society for Cell \& Gene Therapy.

A
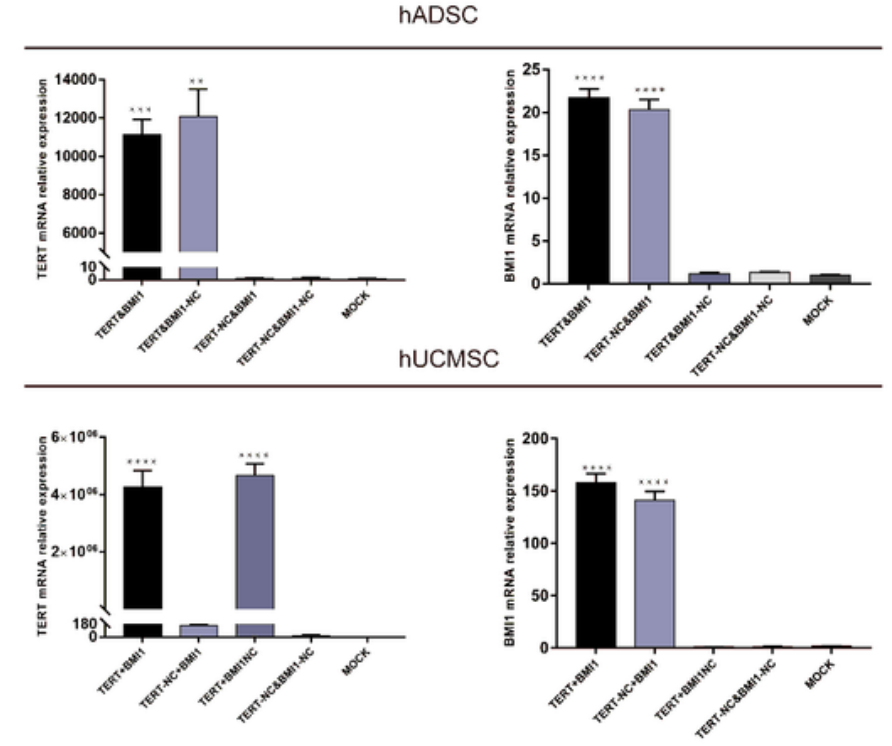

$B$

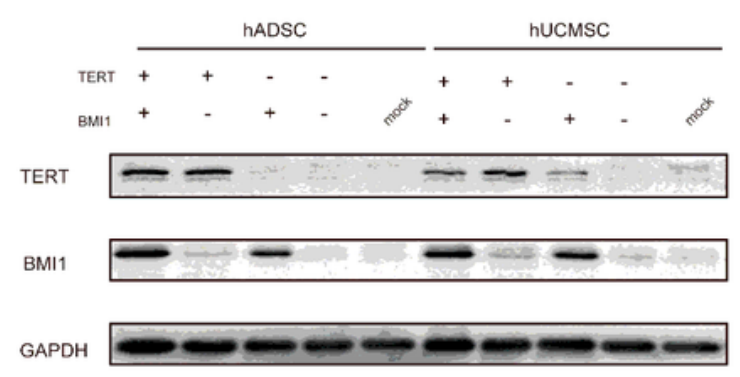

C

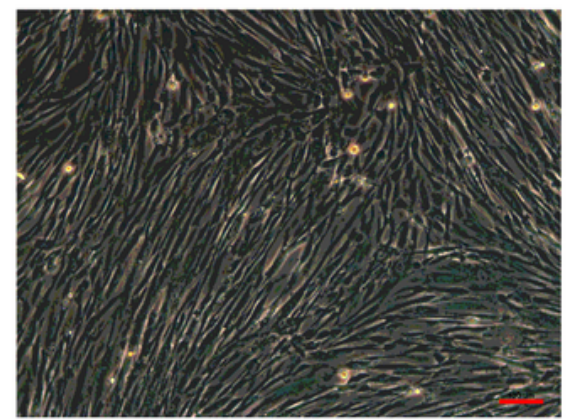

im-hUCMSC

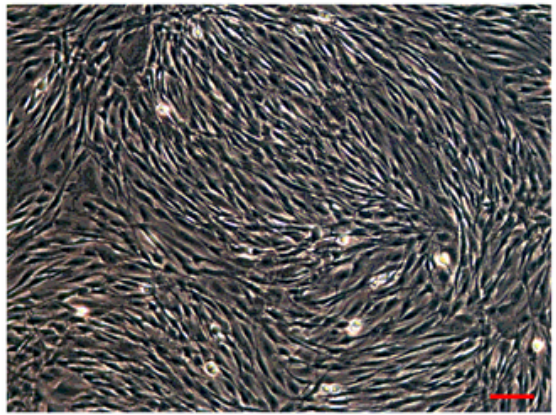

im-hADSC

Figure 2

Protein and mRNA expression of BMI1 and TERT genes in immortalized MSCs. (A) RT-PCR analysis of BMI1 and TERT mRNA expression in hADSC, hUCMSC infected with LV-TERT, LV-BMI1 and control lentivirus. (B) Western blot detection of TERT and BMI1 protein expression in hADSC and hUCMSC after 
overexpressing TERT or BMI1. (C) Microscopic view of immortalized hADSC and hUCMSC, respectively, magnification, $\times 100$, bar $=100 \mathrm{um}$. The results are presented as the mean \pm SD of biological triplicate assays. ${ }^{\star * P}<0.01,{ }^{* \star * P}<0.001,{ }^{\star * \star *} P<0.0001$. LV, lentivirus.

A

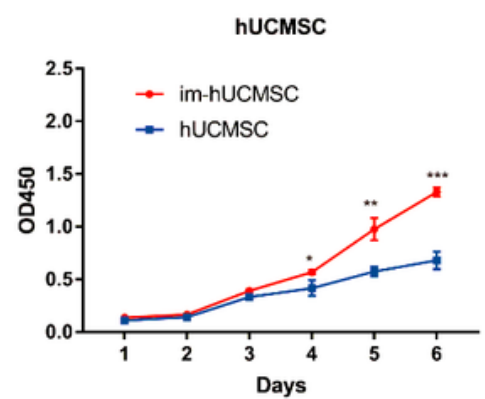

B

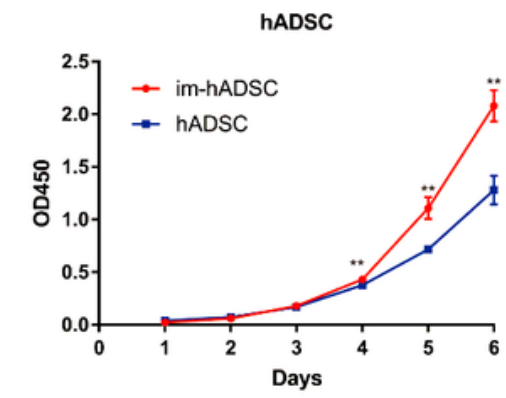

C

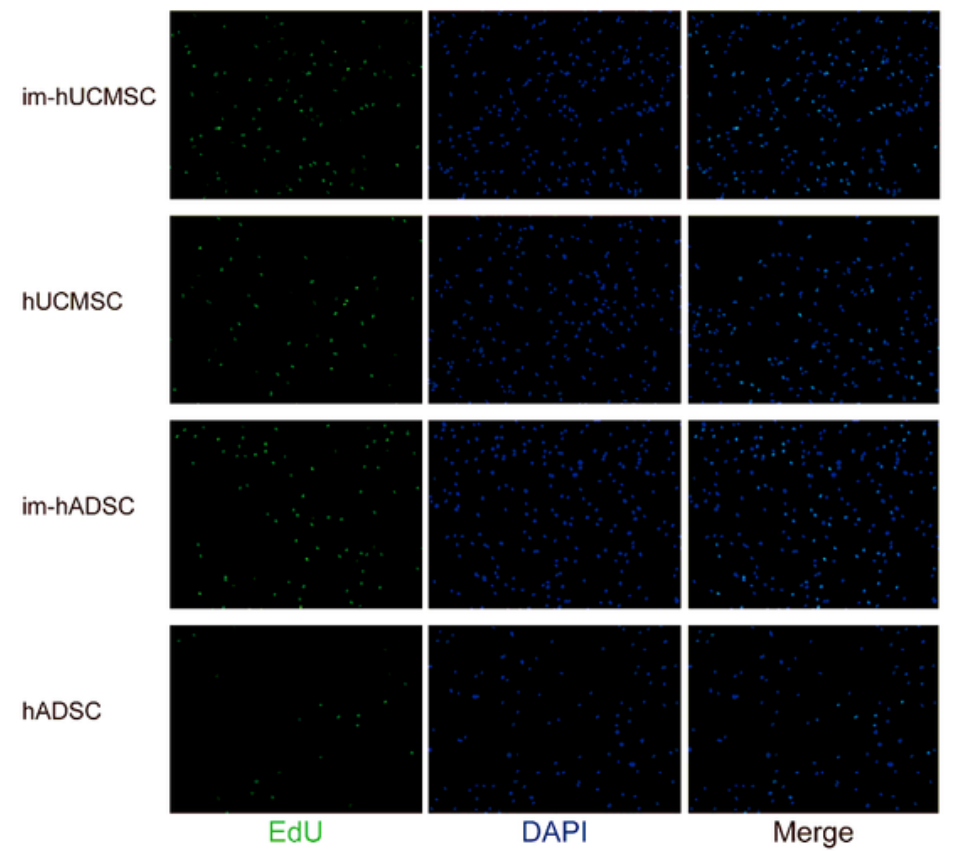

D

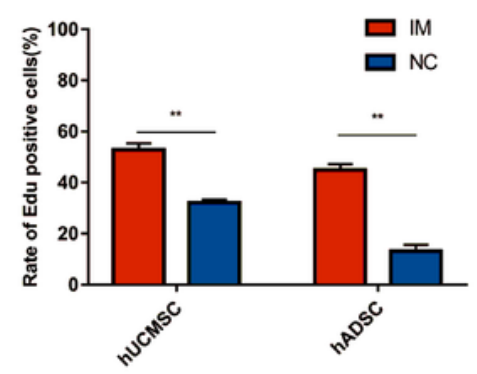

Figure 3

Immortalized mesenchymal stem cells have greater proliferative capacity. (A-B) Cell growth curve of hADSC, hUCMSC and MSCs after transfected by LV-TERT and LV-BMI1, detected by the CCK-8 assay. (C- 
D) By adding EdU reagent, the EdU-positive cells of hADSC, hUCMSC im-hUCMSC and im-hADSC were detected at two hours later magnification, $\times 100$. The rate of EdU-positive cells in hADSC, hUCMSC, imhUCMSC and im-hADSC. The results are presented as the mean \pm SD of biological triplicate assays. ${ }^{*} \mathrm{P}$ $0.05, * \star \mathrm{P}<0.01,{ }^{\star} * \star \mathrm{P}<0.001$. im-hUCMSC, immortalized human umbilical cord mesenchymal stem cells; im-hADSC, immortalized human adipose-derived mesenchymal stem cells.

A

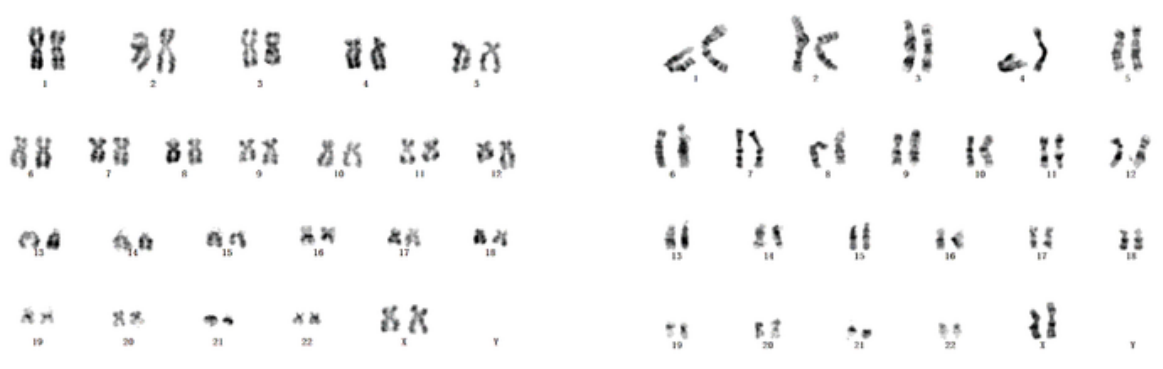

im-hADSC(P55)

im-hUCMSC(P30)

B

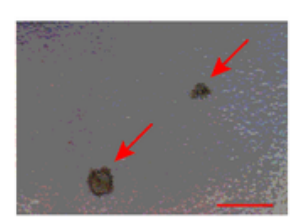

U87

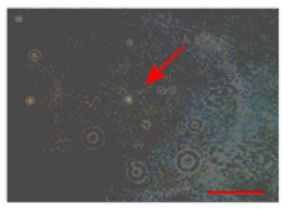

hADSC

C
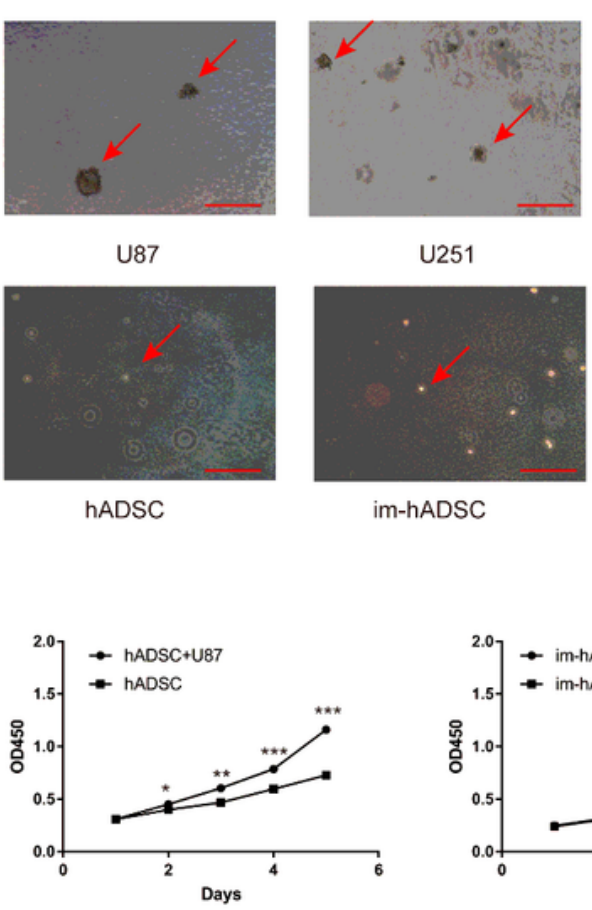

im-hADSC

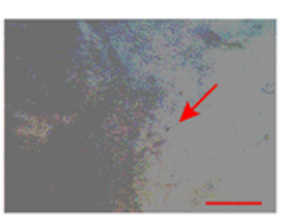

HA

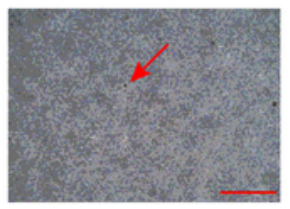

hUCMSC

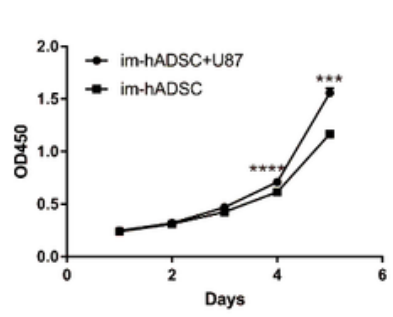

im-hADSC

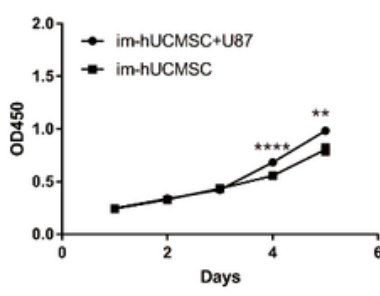

hADSC

hUCMSC

im-hUCMSC

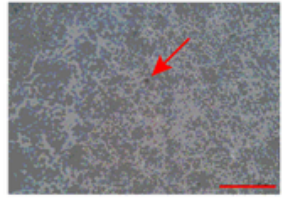

im-hUCMSC

D Co-culture with U87
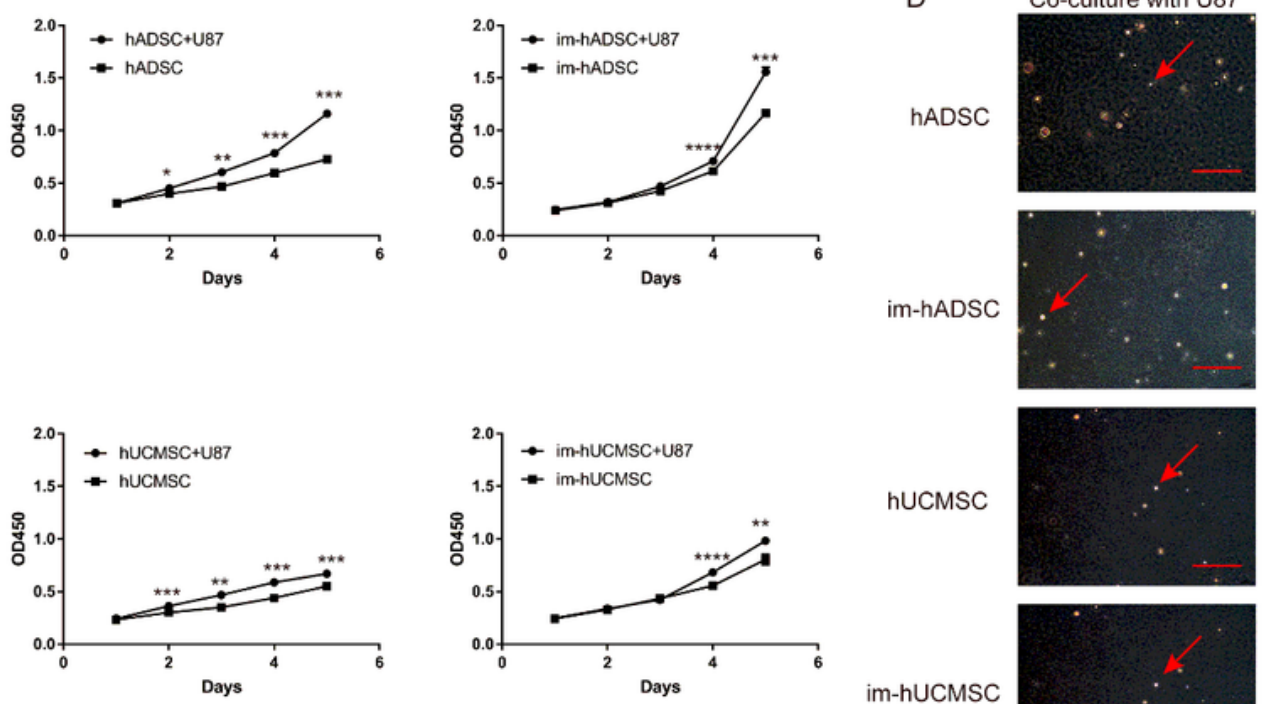

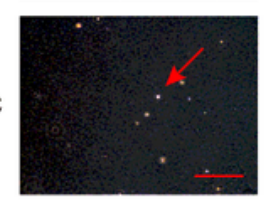

Figure 4 
Safety of immortalized mesenchymal stem cells in vitro. (A) Karyotype analysis of immortalized mesenchymal stem cells. (B) Soft agar clone formation test to detect the clone formation ability of immortalized mesenchymal stem cells, GBM cell lines (U87 and U251) were used as a positive reference, and normal glial cells (HA1800) were used as a negative reference. (C) Cell proliferation curve of mesenchymal stem cells that indirectly co-cultured with U87 cells, detected by the CCK-8 assay. (D) Soft agar clone formation experiment was used to detect the clone formation ability of immortalized mesenchymal stem cells co-cultured with U87 cells indirectly. Magnification, $\times 40$, bar $=500 \mu \mathrm{m} .{ }^{*} \mathrm{P}<0.05$,

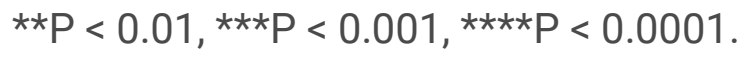


A
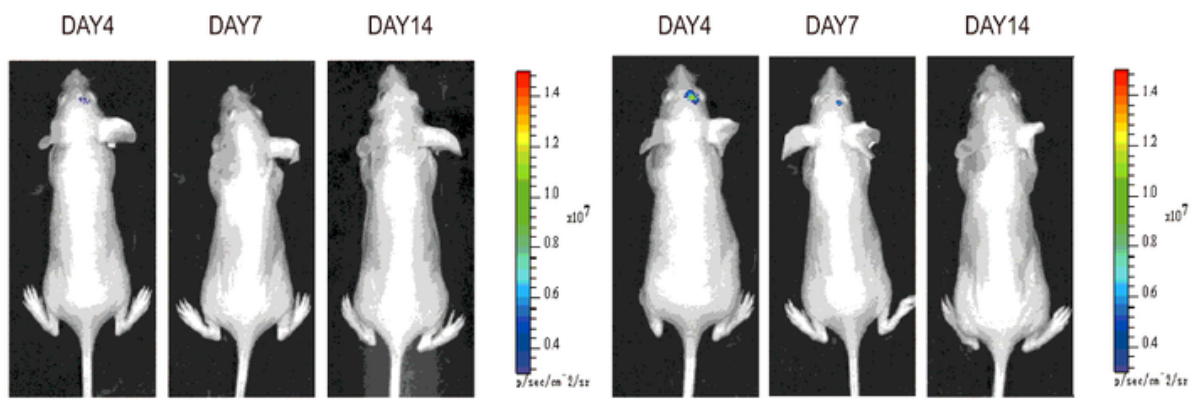

MSC

HA1800
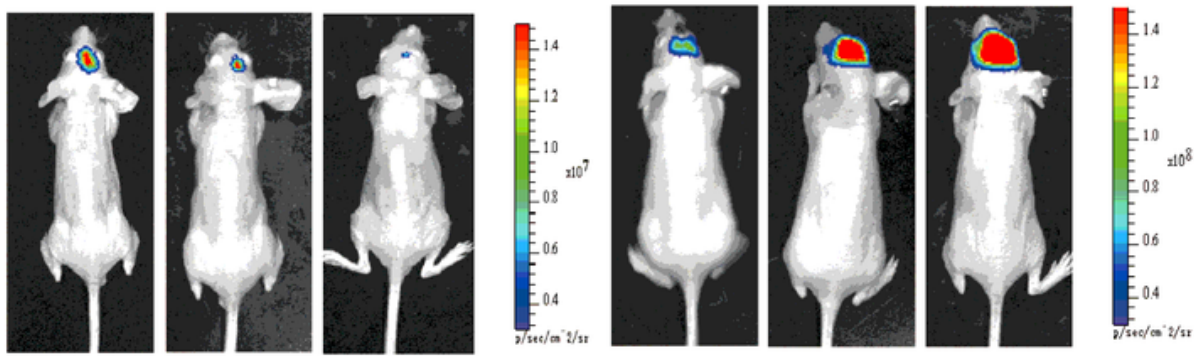

im-MSC

U87

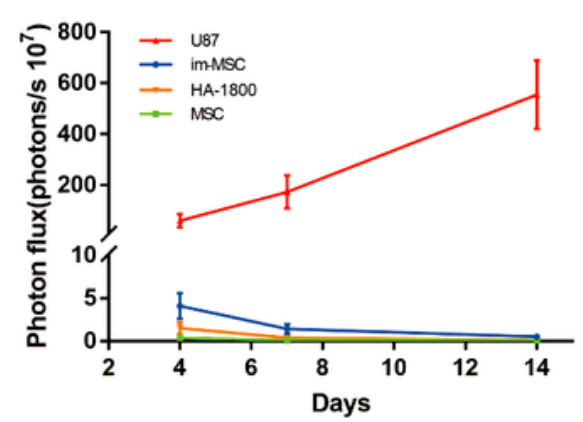

B

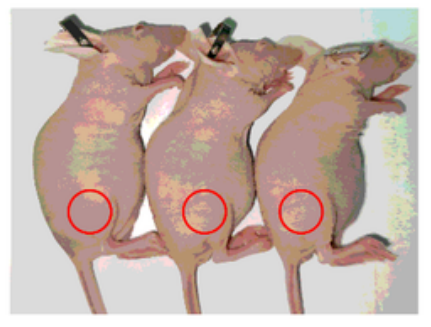

C

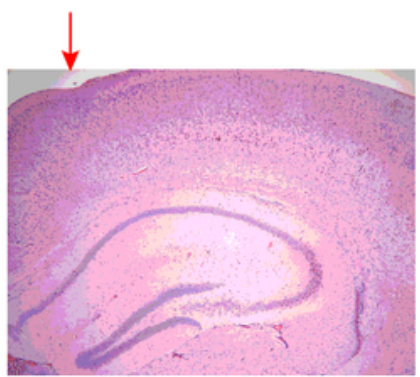

im-MSC

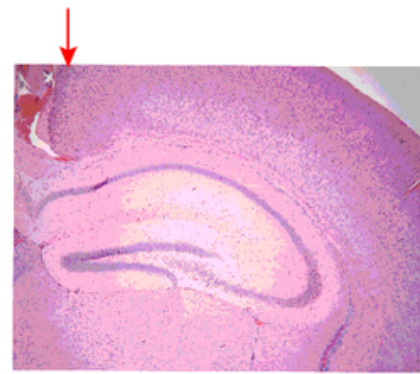

MSC

\section{Figure 5}

Safety of immortalized mesenchymal stem cells in vivo. (A) IVIS luminescent imaging of BALB/c nude mice with intracranial injection of immortalized mesenchymal stem cells $(1 \times 106)$. Line graph showing cell growth in the brain, U87 cells were used as a positive reference, and normal glial cells (HA1800) were used as a negative reference. (B) Im-hMSC cells were injected subcutaneously into the proximal right lower extremity of nude mice to evaluate tumourigenic, the picture was taken on the 14th day after 
injection. (C) HE staining shows that there was no abnormal tissue growth inside the brain where mesenchymal stem cells were injected. Red arrow indicates the direction of needle entry. IVIS, in vivo imaging system; HE staining, hematoxylin-eosin staining.

A

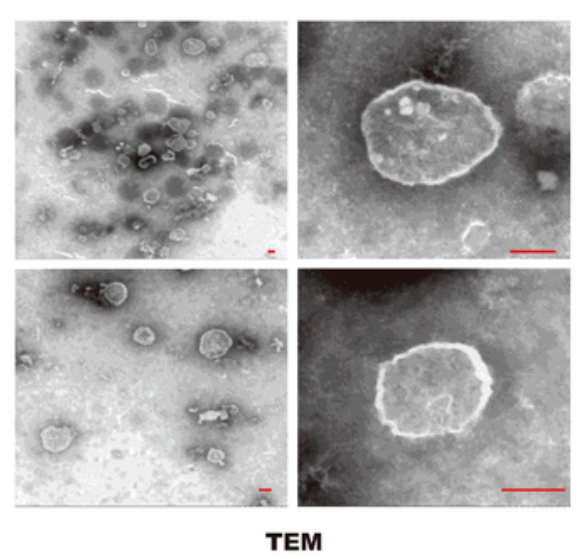

B
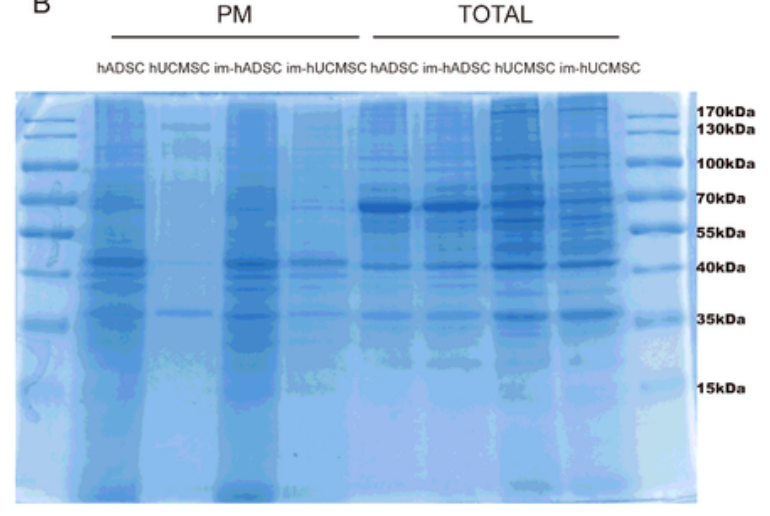

C

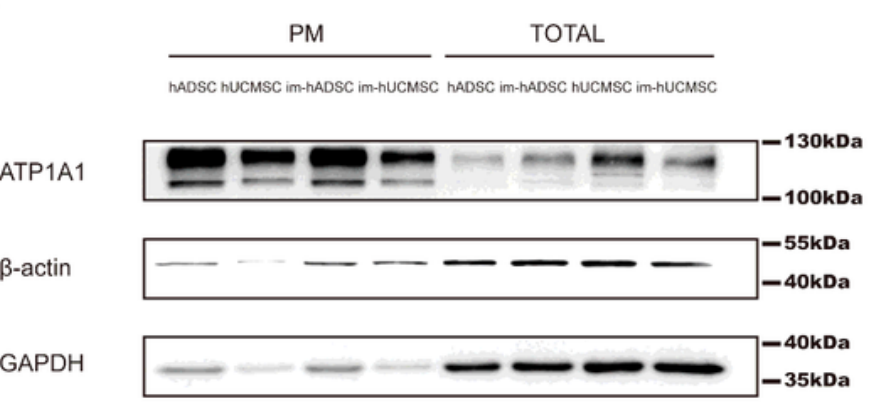

Figure 6

Extraction of Mesenchymal Stem Cell Membrane. (A) TEM image of mesenchymal stem cell membranes under electron microscope. (B) The expression profile of proteins in PM and whole cell lysates of MSCs or 
im-MSCs were analyzed using SDS-PAGE. (C) The expression levels of ATP1A1, $\beta$-actin and GAPDH in PM and whole cell lysates were evaluated using western blot. Scale bar, $100 \mathrm{~nm}$.TEM, transmission electron microscope; PM, plasma membrane.

A

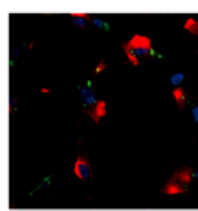

MSC-PM

B

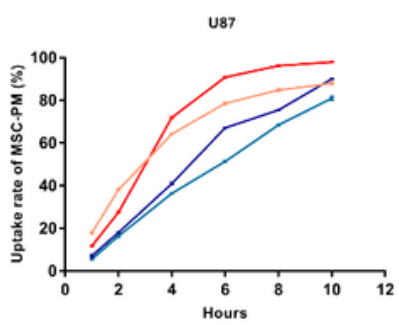

C

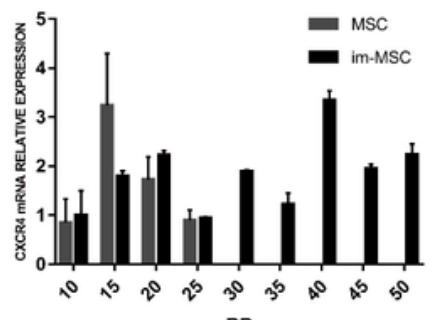

PD

D

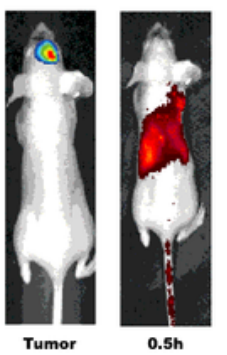

E
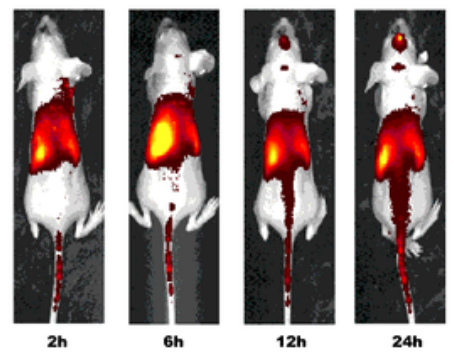

MSC-PM

U251
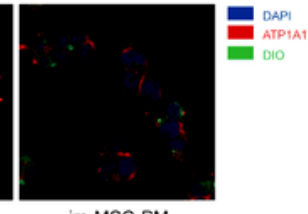

im-MSC-PM
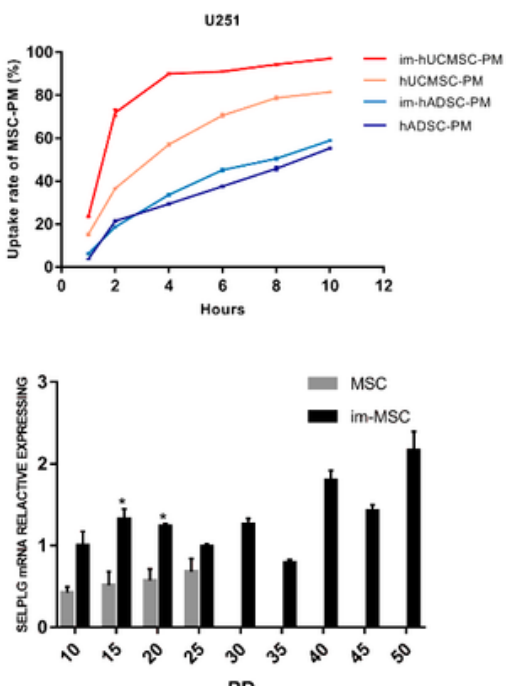

PD
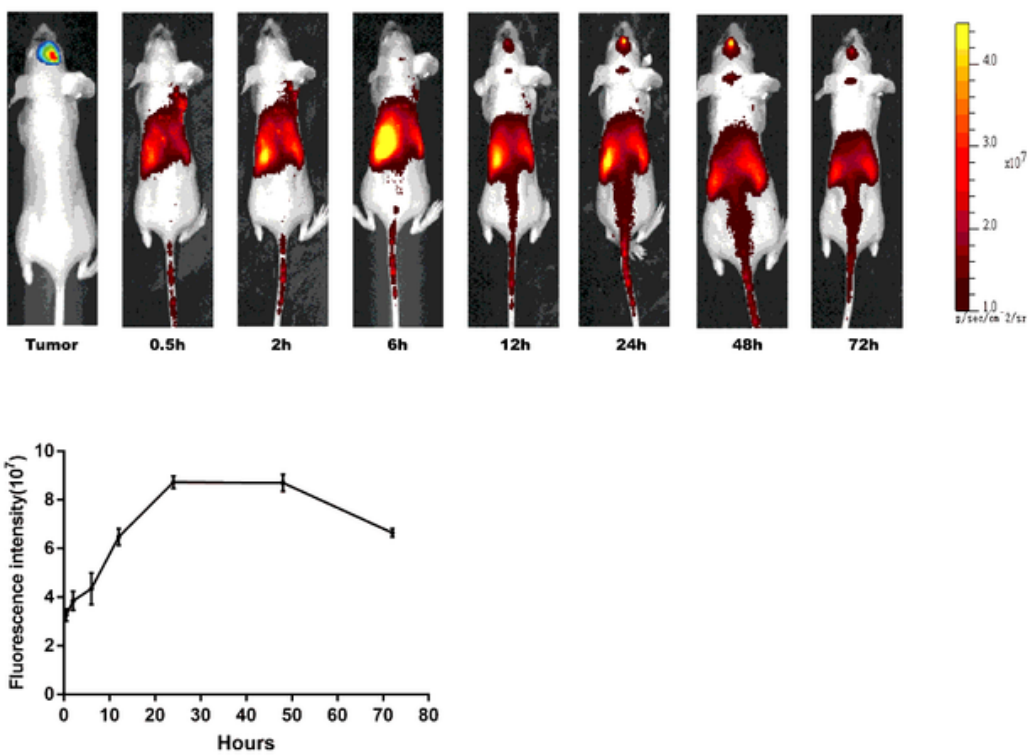

Figure 7

A-C Cellular uptake of PM and expression of tumour-homing molecules. D-E Biodistribution of im-MSCs PM after intravenous (IV) injections. (A) IF staining of ATP1A1 (red) in U87 and U251, PM marked with 
DIO (green), magnification, $\times 200$. (B) Flow cytometry showing the uptake curve of PM by u87 and u251. (C) Realtime reverse transcription-PCR (RT-PCR) detection of CXCR4 and SELPLG mRNA expression in MSCs and im-MSCs of different PDs. The results are presented as the mean \pm SD of biological triplicate assays. ${ }^{*} P<0.05$. (D) Representative IVIS luminescent imaging of mice bearing glioma at different time points $(0.5 \mathrm{~h}, 2 \mathrm{~h}, 6 \mathrm{~h}, 12 \mathrm{~h}, 24 \mathrm{~h}, 48 \mathrm{~h}$, and $72 \mathrm{~h})$. (E) The fluorescence signal intensity of the mice. The results are presented as the mean \pm SD of biological triplicate assays. IF, immunofluorescence; $P D$, population doubling.

\section{Supplementary Files}

This is a list of supplementary files associated with this preprint. Click to download.

- supportingInformation.docx 